\title{
Higher Order Periodic Base Pairs Opening in a Finite Stacking Enthalpy DNA Model
}

\author{
Nkeh Oma Nfor \\ Department of Physics, Higher Teacher Training College Bambili, The University of Bamenda, Bambili, Cameroon \\ Email: omnkeh@gmail.com,nfor.oma@uniba.cm
}

How to cite this paper: Nfor, N.O. (2021) Higher Order Periodic Base Pairs Opening in a Finite Stacking Enthalpy DNA Model. Journal of Modern Physics, 12, 1843-1865. https://doi.org/10.4236/jmp.2021.1213107

Received: October 26, 2021

Accepted: November 26, 2021

Published: November 29, 2021

Copyright $\odot 2021$ by author(s) and Scientific Research Publishing Inc. This work is licensed under the Creative Commons Attribution International License (CC BY 4.0).

http://creativecommons.org/licenses/by/4.0/

\begin{abstract}
The dynamics of periodic base pairs opening in a finite stacking enthalpy DNA is investigated in this work. This is achieved by using the JoyeuxBuyukdagli DNA model, in which the polynomial approximations of the stacking interaction and Morse potential are expanded up to the fifth order nonlinear terms by using the Taylor series expansion technique. By incorporating the continuum limit approximation and the extended multiple scale asymptotic methods, higher order nonlinear Schrödinger amplitude equations are derived. In the limit of cubic nonlinearity, the periodic base pair configurations clearly depict the open state; with linear stability analysis exposing other periodic background modes that are vital in the DNA transcription, replication, and transmission of genetic codes. The higher order modes generally display a more robust and structurally stable wave profile, which epitomizes the base pair dynamics of the DNA molecule observed from experimental investigations. Prolonged time evolution of base pairs stretching greatly modifies the higher order modes of the DNA molecule, strongly suggesting that such modes may induce abnormalities like gene mutation which is responsible for numerous diseases.
\end{abstract}

\section{Keywords}

Base Pairs, Finite Stacking Enthalpy, Transcription, Genetic Codes

\section{Introduction}

The nonlinear dynamics of deoxyribonucleic acid (DNA) remains a very fascinating and active area of research in biophysics. This is because it provides the basis for understanding intrinsic processes like transcription, replication, and 
transmission of genetic codes [1]-[10]. In fact, the DNA is only found in the nucleus of living cells, and has the structure of a double-stranded macromolecule in the form of a double helix [1] [5]. A mastery of the interaction between nucleotides and water molecules is a key factor in understanding the double helix structure.

Concretely, bases are insoluble in water that is hydrophobic, while sugar and phosphate form bonds with water molecules that results to sugar-phosphate backbone. This backbone is generally aligned on the surface of a cylinder, while the bases are oriented toward its center. This configuration leads to a natural protection of the bases which carries most of the genetic codes, by the sugar-phosphate backbone [11]. It is important to note that ribonucleic acid (RNA) links the DNA with protein, hence leading to the effective control of the protein bio-synthesis by the DNA in the transcription process. Experimental results point to the fact that the transcription process is inextricably linked to variation of the DNA environmental temperature [12], during the process of denaturation or melting. The fluid medium that surrounds the DNA equally enhances molecular collisions that may trigger rotational, transverse, and longitudinal oscillations of nucleotides [13] [14].

Solitons are solutions of a widespread class of weakly disperse partial differential equations, and it generally originates from the balance between nonlinearity and dispersion. The soliton concept which emanates from the Fermi Pasta Ulam (FPU) paradox [15] [16], is increasingly being used to explain the complex dynamics of neural networks [17] [18] [19] [20] [21], optical fiber systems [22] [23] [24], and the local base pairs opening of DNA [25] [26]. Concretely, the local base pairs opening of DNA can be analytically captured as breather-like modes of small amplitude. These modes have fascinating properties owing to their small amplitude, like the induction of energy trapping as the breathers move along the DNA strand [27]. Some local dis-homogeneities can equally enhance the trapping mechanism [28], which is indicative of the fact that the properties of breathers could allow the formation of the transcription bubble after the interaction with the bound RNA-polymerase. From the seminal work of Englander et al., the evolution of solitonic excitations in the DNA double chain play crucial roles in the transcription process [29]. Other simplified DNA models include the Y-model introduced by Yakushevich in 1989 [30], which has been improved upon and extensively studied [31] [32] [33]. According to the model, the DNA consists of two parallel chains of discs which are connected to each other with longitudinal and transverse springs. The rigidity of the longitudinal springs is higher than that of the transverse ones as they represent the covalent and hydrogen bonds, respectively. Another interesting model is the Plane-Base Rotator (PBR) model, initially proposed by Yomosa [34] [35] and improved by Homma and Takeno [36]. A degree of freedom characterizing base rotations in the plane perpendicular to the helical axis around the backbone structure is assumed in the PBR model, while the introduced Hamiltonian is based on the 
Heisenberg's spin model for the ferromagnetic chain. The Peyrard-Bishop-Dauxois (PBD) model of DNA remains a very successfully model to analyze experiments on short DNA sequences [37], and able to mimic real denaturation curves identified by Raman spectroscopy [8]. The PBD model incorporates stacking interactions between neighboring base pairs to enhance the rigidity of secondary DNA structure. However, it should be noted that this stacking interaction inherent in the PBD model does not associate any characteristic energy in the important dynamics of the DNA system [8].

The quest to incorporate finite characteristic energies with phase transition triggered numerous research activities on DNA dynamics; tailored on capturing the appropriate phase transition predicted by statistical DNA models. This finally culminated with the brilliant works of Joyeux and Buyukdagli (BJ) model of DNA [2] [3] [4], which is based on site-specific stacking enthalpies. This model is very reliable because it reproduced exact experimental curves that ensured a sharp melting transition, as a result of the finiteness of the stacking interaction [2] [3] [4]. Carlos et al. exploited the JB model to investigate on the dynamics of discrete breathers which is governed by the extended discrete nonlinear Schrödinger equation [38]. Depending on the finite stacking parameters, compact bright solitary waves became more robust or quickly decomposed in the JB model of DNA [39]. On the other hand, Ying-Bo Yao et al. demonstrated that the JB system is capable of producing high-order envelope solitons; which can be viewed as high-order discrete breathers with zero group velocity at the center of the Brillouin zone [26].

Unlike the aforementioned studies which deal with spatially localized excitations, the present investigation seeks to explore periodic solutions that may characterize base pairs opening. Periodic wave train solutions gives a better understanding of myriad of bio-physical activities hitherto explained only by localized solutions. For example, Vargas et al. numerically exploited localized periodic solutions in the nerve model, to rigorously explain hyper-polarization, pulse trains, and refractory periods that were experimentally observed in the nerve of locust [18] [40]. Also, the energy released during the hydrolysis of adenosine triphosphate was shown to be transported via periodic soliton wave train in order to sustain important biological processes like enzyme catalysis and muscle contractions [41]. From a physiological standpoint, gene expression is very important in life because genetic codes are regularly transferred during protein synthesis [3] [5]. The effective stimulation of all the base pairs is essential for the DNA loop formation [38], regulation of gene expressions, and packaging of DNA into nucleosomes [5]. In fact, the RNA which is a key component in the transfer, transcription, and messaging in DNA, operates in few portions of the DNA sequence at the same time [5]. Such complex motion strongly suggests that it is more appropriate for the DNA base pairs opening to be considered as a spatial periodic activity, in order to holistically comprehend the DNA dynamics. We are therefore interested on how changes in the stacking parameters can generate new spa- 
tial periodic base pairs opening profiles, that may be responsible for some physiological abnormalities. Such open states are vulnerable to many external attacks that may cause reading or coding errors, which is responsible for numerous cancers induced by gene mutations [25] [42]. To the best of my knowledge, this is one of the rare studies that seek to model base pairs opening as a spatial periodic phenomenon.

In the present work, we demonstrate that the BJ model of DNA supports spatial periodic base pairs open configurations. Consequently, the organization of the paper is structured as follows. In section 2, we present the Hamiltonian of the $\mathrm{BJ}$ model which naturally leads us to the discrete equations of motion for the in-phase and out-of-phase motions, respectively. We expand terms of the stacking interaction and Morse potential in the out-of-phase equation of motion to the fifth order and implore the continuum limit approximation. Higher order amplitude equations are derived by using the extended multiple-scales asymptotic perturbation method. Analysis in section 3 is limited to terms up to the cubic order, in which periodic base pairs opening are captured to describe the open state configurations of the DNA. Stability analysis of these open states further reveal other localized background modes that are crucial in the transcription process. In section 4 , we further explore higher order modes of the DNA open states configuration. This gives us a better opportunity to analytically and numerically investigate on the richer dynamics of DNA. Finally in section 5, we will summarize the important results obtained and articulate on some brighter perspectives.

\section{Model and Equations of Motion}

The dynamical DNA model proposed by Joyeux and Buyukdagli is more realistic than the PBD model. This is because the JB model is based on site-specific stacking enthalpies, with the finiteness of the stacking interaction sufficiently ensuring a sharp melting transition [2] [3] [4]. The DNA molecule in the JB model is considered as two elastic chains of nucleotides, that represent the double helix strand of the molecule. The nucleotides in the same strand experience nearest-neighbor interactions along the one dimensional chain configuration. The molecule is assumed homogeneous, with each strand linked to the other by hydrogen bonds which are modeled by the Morse potential. The longitudinal, rotational, and torsional motions of the DNA base pairs are all ignored, with focus only on the transverse motions. Concretely, this transverse displacements from the equilibrium position of the nucleotide pairs located in opposite strands are given by $x_{n}$ and $y_{n}$. This naturally leads to the Hamiltonian of the BJ model as [2] [3]

$$
H=\sum_{n}\left\{\frac{1}{2} m\left(\dot{x}_{n}^{2}+\dot{y}_{n}^{2}\right)+V_{n}\left(x_{n}, y_{n}\right)+W_{n}\left(x_{n}, y_{n}, x_{n-1}, y_{n-1}\right)\right\},
$$

where 


$$
\begin{aligned}
V_{n}\left(x_{n}, y_{n}\right) & =D_{0}\left[\mathrm{e}^{-a\left(x_{n}-y_{n}\right)}-1\right]^{2} \\
W_{n}\left(x_{n}, y_{n}, x_{n-1}, y_{n-1}\right)= & \frac{\Delta H_{n}}{C}\left[2-\mathrm{e}^{-b\left(x_{n}-x_{n-1}\right)^{2}}-\mathrm{e}^{-b\left(y_{n}-y_{n}\right)^{2}}\right] \\
& +K_{b}\left[\left(x_{n}-x_{n-1}\right)^{2}+\left(y_{n}-y_{n-1}\right)^{2}\right] .
\end{aligned}
$$

Each base pair position is represented by $n$ with $N$ being the total number of the base pairs of the chain, while $m$ is the average mass of the nucleotides. The on-site potential $V_{n}\left(x_{n}, y_{n}\right)$ is due to the presence of hydrogen bonds which is described by the Morse potential of depth $D_{0}$ and width $a$. The Morse potential opposes the breaking of the hydrogen bonds because it is an increasing function of the distance between the two bases of a pair $n$. The first term in the potential $W_{n}\left(x_{n}, y_{n}, x_{n-1}, y_{n-1}\right)$ describes the finite stacking interaction, while the second one models the stiffness of the sugar-phosphate backbone. Both terms are increasing functions of $\left|x_{n}-x_{n-1}\right|$ (as well as $\left|y_{n}-y_{n-1}\right|$ ), which implies that they oppose the de-stacking of the bases. The stacking potential which is approximated by a Gaussian hole of depth $\frac{\Delta H_{n}}{C}$, emanates from hydrophobic interactions with the solvent and electronic interactions between successive base pairs on the same strand. The backbone stiffness is taken as a harmonic potential of constant $K_{b}$, ensures that base pairs belonging to the same strand do not separate infinitely when approaching the melting temperature.

It is more convenient to introduce the coordinates $u_{n}$ describing the movement of a center of mass of the nucleotide pair, and $v_{n}$, a stretching of the nucleotides belonging to the same pair defined as

$$
u_{n}=\frac{x_{n}+y_{n}}{\sqrt{2}}, \quad \text { and } \quad v_{n}=\frac{x_{n}-y_{n}}{\sqrt{2}}
$$

The in-phase motion is actually governed by $u_{n}$, while $v_{n}$ represent the out-of-phase motion. From the Hamiltonian (1), it is possible to obtain two nonlinear discrete differential equations describing the transverse in-phase and out-of-phase dynamics of the DNA molecular chain respectively given as [25]

$$
\begin{aligned}
\ddot{u}_{n}= & \frac{2 K_{b}}{m}\left[\left(u_{n+1}-u_{n}\right)+\left(u_{n-1}-u_{n}\right)\right] \\
& +\frac{2 b \Delta H_{n}}{m C}\left[\left(u_{n+1}-u_{n}\right) \mathrm{e}^{-b\left(u_{n+1}-u_{n}\right)^{2}}+\left(u_{n-1}-u_{n}\right) \mathrm{e}^{-b\left(u_{n-1}-u_{n}\right)^{2}}\right], \\
\ddot{v}_{n}= & \frac{2 K_{b}}{m}\left[\left(v_{n+1}-v_{n}\right)+\left(v_{n-1}-v_{n}\right)\right]+\frac{2 b \Delta H_{n}}{m C}\left[\left(v_{n+1}-v_{n}\right) \mathrm{e}^{-b\left(v_{n+1}-v_{n}\right)^{2}}\right. \\
- & \left.\left(v_{n-1}-v_{n}\right) \mathrm{e}^{-b\left(v_{n-1}-v_{n}\right)^{2}}\right]+\frac{2 \sqrt{2} a D_{0}}{m} \mathrm{e}^{-a \sqrt{2} v_{n}}\left[\mathrm{e}^{-a \sqrt{2} v_{n}}-1\right] .
\end{aligned}
$$

From a comparative analysis between Eqns (3a) and (3b), it is clear that the effects of nonlinearity are more pronounced in the out-of-phase motion Equation (3b). This is because Equation (3b) which mimics base pair stretching, incorporates hydrogen bonds interactions which is a vital component in the DNA 
dynamics. On the other hand the in-phase equation of motion (3a), is more associated with strong covalent bonds with little or no effects on the holistic DNA dynamics [25]. Hence forth, we will neglect Equation (3a) and deal only with the out-of-phase motion Equation (3b), and adopt the following experimental parameters [2] [4]: $m=300 \mathrm{amu}, D_{0}=0.04 \mathrm{eV}, a=4.45 \AA^{-1}, \Delta H_{n}=0.44 \mathrm{eV}$, $b=0.10 \AA^{-2}, K_{b}=10^{-5} \mathrm{eV} \cdot \AA^{-2}$, and $C=2.00$. It is further assumed that the base pair oscillations are quite large enough to induce inharmonicity, but still inadequate to destroy the hydrogen bonds because the plateau of the Morse potential is not attained. Based on this assumption, the base nucleotides should oscillate around the bottom of the Morse potential. Consequently one can expand the exponential functions up to the fifth order in the Taylor series approximation, and rewrite Equation ( $3 \mathrm{~b}$ ) as

$$
\begin{aligned}
\ddot{v}_{n}= & \frac{2}{m}\left(K_{b}+\frac{b \Delta H_{n}}{C}\right)\left(v_{n+1}-2 v_{n}+v_{n-1}\right) \\
& -\frac{2 b^{2} \Delta H_{n}}{m C}\left[\left(v_{n+1}-v_{n}\right)^{3}-\left(v_{n}-v_{n-1}\right)^{3}\right] \\
& +\frac{b^{3} \Delta H_{n}}{m C}\left[\left(v_{n+1}-v_{n}\right)^{5}-\left(v_{n}-v_{n-1}\right)^{5}\right] \\
& -\frac{2 a^{2} D_{0}}{m}\left[v_{n}-\frac{3 a}{2} v_{n}^{2}+\frac{7 a^{2}}{6} v_{n}^{3}-\frac{15 a^{3}}{24} v_{n}^{4}+\frac{31 a^{4}}{120} v_{n}^{5}\right] .
\end{aligned}
$$

The discrete coupled nonlinear Equation (4) is non integrable, but an approximation can be implored which preserves the nonlinearity of the system and reduce Equation (4) to an integrable form of a partial differential equation in order to obtain analytic solutions. Let us assume that the base pairs stretching $v_{n}(t)$ changes only slightly from one site to the next such that $v_{n}(t)=v(z=n r, t)$, where $z$ is a dimensionless variable that measures the position along the DNA strand. $r$ is a measure of the equilibrium distance between two successive neighboring nucleotides in the same strand, with numerical value of $3.4 \AA$ in a real DNA molecule [43]. Hence by considering a slow spatial variation of $v(z, t)$, and exploiting a Taylor expansion around $z=n r$, leads to

$$
v_{n \pm 1}(t) \approx v \pm r \frac{\partial v}{\partial z}+\frac{r^{2}}{2} \frac{\partial^{2} v}{\partial z^{2}}+\cdots
$$

The continuum limit approximation (5), transforms Equation (4) to

$$
\begin{aligned}
\frac{\partial^{2} v}{\partial t^{2}}= & {\left[\frac{2 r^{2}}{m}\left(K_{b}+\frac{b \Delta H_{n}}{C}\right)+\frac{6 r^{4} b^{2} \Delta H_{n}}{m C}\left(\frac{\partial v}{\partial z}\right)^{2}+\cdots\right] \frac{\partial^{2} v}{\partial z^{2}} } \\
& -\frac{2 a^{2} D_{0}}{m}\left[v-\frac{3 a}{2} v^{2}+\frac{7 a^{2}}{6} v^{3}-\frac{5 a^{3}}{8} v^{4}+\frac{31 a^{4}}{120} v^{5}\right],
\end{aligned}
$$

with all the key features involved in the DNA dynamics maintained, and terms of order $\mathrm{O}\left(r^{5}\right)$ or higher are neglected. It is more convenient to transform Equation (6) into a an appealing form by considering the dimensionless variables [38] [39] 


$$
\begin{aligned}
& V=a v, t=\sqrt{\frac{m}{a^{2} D_{0}}} \tau, K_{0}=\left(\frac{2 r^{2}}{a^{2} D_{0}}\right)\left(K_{b}+\frac{b \Delta H_{n}}{C}\right), K_{1}=\frac{6 r^{4} b^{2} \Delta H_{n}}{a^{4} C D_{0}}, \\
& \omega^{2}=2, \alpha=3 / 2, \beta=-7 / 6, \gamma=5 / 8, \sigma=-31 / 120 .
\end{aligned}
$$

Equation (6) is now written in the dimensionless form

$$
\frac{\partial^{2} V}{\partial \tau^{2}}=\left[K_{0}+K_{1}\left(\frac{\partial V}{\partial z}\right)^{2}+\cdots\right] \frac{\partial^{2} V}{\partial z^{2}}-\omega^{2}\left[V-\alpha V^{2}-\beta V^{3}-\gamma V^{4}-\sigma V^{5}\right],
$$

in which in the new dimensionless time unit, we have that $\tau=1.00$, corresponds to $t=0.198 \mathrm{ps}$.

To find the solution of Equation (8), we must first obtain a useful and manageable equation by assuming a more appropriate ansatz; which we postulate that the asymptotic series is more generalized while preserving the essential features of the DNA system. This can be done using reductive perturbative analysis, which is more robust in that it can work for a wide range of problems. In fact, we explore the extended multiple-scales asymptotic approach to reduce Equation (8) to higher order nonlinear Schrödinger amplitude equations. The main idea behind the asymptotic approach is to introduce fast and slow time and spatial variables into Equation (8), by exploiting the perturbation parameter $\varepsilon \ll 1$. The hierarchies of new independent variables replacing $z$ and $\tau$ are

$$
\left\{\begin{array}{l}
t_{0}=\tau, t_{2}=\varepsilon^{2} \tau, t_{4}=\varepsilon^{4} \tau, \cdots \\
z_{1}=\varepsilon z, z_{3}=\varepsilon^{3} z, \cdots
\end{array}\right.
$$

so that the $\tau$ - and $z$-derivatives are replaced by

$$
\begin{gathered}
\frac{\partial}{\partial \tau}=\frac{\partial}{\partial t_{0}}+\varepsilon^{2} \frac{\partial}{\partial t_{2}}+\varepsilon^{4} \frac{\partial}{\partial t_{4}}+\cdots, \\
\frac{\partial}{\partial z}=\varepsilon \frac{\partial}{\partial z_{1}}+\varepsilon^{3} \frac{\partial}{\partial z_{3}}+\cdots .
\end{gathered}
$$

According to the extended reductive perturbation method, we consider the following ansatz for the solution of $V(z, \tau)$ as [44]

$$
\begin{aligned}
V(z, \tau)= & \varepsilon\left(\psi \mathrm{e}^{i \omega t_{0}}+\psi^{*} \mathrm{e}^{-i \omega t_{0}}\right)+\varepsilon^{2}\left(G_{2}+H_{2} \mathrm{e}^{2 i \omega t_{0}}+H_{2}^{*} \mathrm{e}^{-2 i \omega t_{0}}\right) \\
& +\varepsilon^{3}\left(F_{3} \mathrm{e}^{i \omega t_{0}}+F_{3}^{*} \mathrm{e}^{-i \omega t_{0}}+J_{3} \mathrm{e}^{3 i \omega t_{0}}+J_{3}^{*} \mathrm{e}^{-3 i \omega t_{0}}\right) \\
& +\varepsilon^{4}\left(G_{4}+H_{4} \mathrm{e}^{2 i \omega t_{0}}+H_{4}^{*} \mathrm{e}^{-2 i \omega t_{0}}+K_{4} \mathrm{e}^{4 i \omega t_{0}}+K_{4}^{*} \mathrm{e}^{-4 i \omega t_{0}}\right),
\end{aligned}
$$

where the amplitudes $\psi, G_{2}, H_{2}, F_{3}, J_{3}, G_{4}, H_{4}, K_{4}$ and their complex conjugates $\psi^{*}, H_{2}^{*}, F_{3}^{*}, J_{3}^{*}, H_{4}^{*}, K_{4}^{*}$ are functions of $\left(z_{1}, t_{2}, z_{3}, t_{4}\right)$, while $\omega$ stands for the angular frequency. Upon substitution of the ansatz (10) into the DNA Equation (8) yields a series of inhomogeneous equations at different orders of $\left[\varepsilon, \mathrm{e}^{i \omega t_{0}}\right]$.

Grouping terms to orders $\left[\varepsilon^{2}, \mathrm{e}^{0 i \omega t_{0}}\right],\left[\varepsilon^{2}, \mathrm{e}^{2 i \omega t_{0}}\right]$, and $\left[\varepsilon^{3}, \mathrm{e}^{i \omega t_{0}}\right]$, respectively yields the equations

$$
\begin{gathered}
0=-\omega^{2} G_{2}+\omega^{2} \alpha|\psi|^{2}, \\
-4 \omega^{2} H_{2}=-\omega^{2} H_{2}+\omega^{2} \alpha \psi^{2},
\end{gathered}
$$




$$
\begin{aligned}
& 2 i \omega \frac{\partial \psi}{\partial t_{2}} \\
& =K_{0} \frac{\partial^{2} \psi}{\partial z_{1}^{2}}+2 \alpha \omega^{2}\left[\psi G_{2}+\psi^{*} H_{2}+\psi G_{2}^{*}\right]+3 \beta \omega^{2}|\psi|^{2} \psi .
\end{aligned}
$$

Simplification of Equation (11a) gives $G_{2}=\alpha|\psi|^{2}$, while that of Equation (11b) result in $H_{2}=-\alpha \psi^{2} / 3$, and Equation (11c) is eventually reduced to

$$
i \frac{\partial \psi}{\partial t_{2}}-P \frac{\partial^{2} \psi}{\partial z_{1}^{2}}+Q|\psi|^{2} \psi=0
$$

where

$$
\left\{\begin{array}{l}
P=\frac{r^{2}}{a^{2} \omega D_{0}}\left[K_{b}+\frac{b \Delta H_{n}}{C}\right], \\
Q=-\frac{\omega}{6}\left[10 \alpha^{2}+9 \beta\right] .
\end{array}\right.
$$

The dispersion coefficient $P$, and nonlinear coefficient $Q$, are inextricably linked to the intrinsic constants $r, a, \omega, D_{0}, K_{b}, b, \Delta H_{n}, C, \alpha, \beta$, which are vital in the DNA dynamics; because it determines the type of wave profile in the system. The NLS Equation (12) is a prominent equation used to model a plethora of weakly nonlinear quasi-harmonic wave packets, like the propagation of electromagnetic waves in optical fibers [22] [23] [24], and DNA base pairs opening [25] [26].

This work is quite unique because it considers higher order modes of the DNA dynamics by incorporating the terms $F_{3}, J_{3}, G_{4}, H_{4}, K_{4}$, which highly depend on the type of solution emanating from Equation (12). Consequently, terms to orders $\left[\varepsilon^{3}, \mathrm{e}^{3 i \omega_{0}}\right],\left[\varepsilon^{4}, \mathrm{e}^{0 i \omega t_{0}}\right]$, and $\left[\varepsilon^{4}, \mathrm{e}^{2 i \omega t_{0}}\right]$, respectively gives

$$
\begin{aligned}
& -9 \omega^{2} J_{3}=-\omega^{2} J_{3}+2 \alpha \omega^{2} \psi H_{2}+\beta \omega^{2} \psi^{3}, \\
0= & K_{0}\left(\frac{\partial^{2} G_{2}}{\partial z_{1}^{2}}+\frac{\partial^{2} G_{2}^{*}}{\partial z_{1}^{2}}\right)-\omega^{2}\left(G_{4}+G_{4}^{*}\right)+6 \gamma \omega^{2}|\psi|^{4} \\
+ & \alpha \omega^{2}\left[2 \psi F_{3}^{*}+2 \psi^{*} F_{3}+\left(G_{2}+G_{2}^{*}\right)^{2}+2\left|H_{2}\right|^{2}\right] \\
+ & 3 \beta \omega^{2}\left[\psi^{2} H_{2}^{*}+\psi^{* 2} H_{2}+2|\psi|^{2}\left(G_{2}+G_{2}^{*}\right)\right], \\
-4 \omega^{2} H_{4}+ & 4 i \omega \frac{\partial H_{2}}{\partial t_{2}}= \\
& K_{0} \frac{\partial^{2} H_{2}}{\partial z_{1}^{2}}-\omega^{2} H_{4}+4 \gamma \omega^{2}|\psi|^{2} \psi^{2} \\
& +3 \beta \omega^{2}\left[\psi^{2}\left(G_{2}+G_{2}^{*}\right)+2|\psi|^{2} H_{2}\right] \\
& +\alpha \omega^{2}\left[2 \psi F_{3}+2 \psi^{*} J_{3}+2 G_{2} H_{2}+2 G_{2}^{*} H_{2}\right] .
\end{aligned}
$$

Finally, grouping terms to orders $\left[\varepsilon^{4}, \mathrm{e}^{4 i \omega t_{0}}\right]$ and $\left[\varepsilon^{5}, \mathrm{e}^{i \omega t_{0}}\right]$, respectively generates the equations

$$
-16 \omega^{2} K_{4}=-\omega^{2} K_{4}+\alpha \omega^{2} H_{2}^{2}+2 \alpha \omega^{2} \psi J_{3}+\gamma \omega^{2} \psi^{4}+3 \beta \omega^{2} \psi^{2} H_{2},
$$

and 


$$
\begin{aligned}
& \frac{\partial^{2} \psi}{\partial t_{2}^{2}}+2 i \omega \frac{\partial \psi}{\partial t_{4}}+2 i \omega \frac{\partial F_{3}}{\partial t_{2}} \\
& =K_{0} \frac{\partial^{2} F_{3}}{\partial z_{1}^{2}}+2 K_{0} \frac{\partial^{2} \psi}{\partial z_{1} \partial z_{3}}+10 \sigma \omega^{2}|\psi|^{4} \psi \\
& +4 \gamma \omega^{2}\left[\psi^{3} H_{2}^{*}+3|\psi|^{2} \psi\left(G_{2}+G_{2}^{*}\right)+3|\psi|^{2} \psi^{*} H_{2}\right]+3 \beta \omega^{2}\left[\psi\left(G_{2}+G_{2}^{*}\right)^{2}\right. \\
& \left.+2 \psi^{*} H_{2}\left(G_{2}+G_{2}^{*}\right)+2\left|H_{2}\right|^{2} \psi+\psi^{2^{*}} J_{3}+\psi^{2} F_{3}^{*}+2|\psi|^{2} F_{3}\right] \\
& +2 \alpha \omega^{2}\left[\psi\left(G_{4}+G_{4}^{*}\right)+\psi^{*} H_{4}+F_{3}^{*} H_{2}+F_{3}\left(G_{2}+G_{2}^{*}\right)+H_{2}^{*} J_{3}\right] .
\end{aligned}
$$

Equations (12) to (17) will be used to comprehensively study periodic base pair opening in a DNA double strand, in order to give satisfactory explanations to the transcription and replication processes from a biophysical perspective. However the analysis in section III will be limited just to Equation (12), in which we focus only on the periodic DNA dynamics in the cubic limit [2]-[8] [14]. The higher order modes that deals with Equations (13) to (17), is quite innovative and will be considered separately in section 4 .

\section{Dynamics of Base Pairs Opening in the Cubic Limit}

\subsection{Periodic Solution of the Nonlinear Schrödinger Amplitude Equation}

We now consider the profile of the solution dictated by the derived amplitude Equation (12). Based on experimental parameters [2] [4], it is very clear that the dispersion coefficient $P>0$, and nonlinear coefficient $Q<0$. Consequently the system of Equation (13) can only support bright solitons as a result of the process of modulational instability [14] [15] [17] [19] [20] [21], because $P Q<0$. Plane waves gradually evolve into nonlinear periodic modes, that leads to energy activation in a DNA double strand chain by the process of modulational instability (MI). The MI process equally leads to the spontaneous emission of breather-like modes, and generally thrives in a DNA chain as a result of the dynamic interplay between nonlinearity (emanating from the hydrogen bonds) and dispersion (induced by stacking interaction and sugar-phosphate backbone stiffness).

In order to look for stationary solutions to Equation (12), we assume an ansatz of the form

$$
\psi\left(z_{1}, t_{2}\right)=a\left(z_{1}\right) \mathrm{e}^{i b t_{2}},
$$

where $b$ is the modulation frequency of the envelope and $a\left(z_{1}\right)$ is a real constant that represent the amplitude of the field envelope. Upon substituting Equation (18) into Equation (12) gives

$$
-b a-P \frac{\partial^{2} a}{\partial z_{1}^{2}}+Q a^{3}=0
$$

which can be conveniently transformed to a first-order integral equation 


$$
\left(\frac{\mathrm{d} a}{\mathrm{~d} z_{1}}\right)^{2}=-\frac{b}{P} a^{2}+\frac{Q}{2 P} a^{4}+C
$$

It is important to note that the integration constant $C$ is a key parameter that determines the nature of solution of the amplitude $a\left(z_{1}\right)$. For a localized profile solution in which $a\left(z_{1}\right)$ vanishes as $z_{1} \rightarrow \pm \infty, C$ naturally turns to zero. Consequently the solution to Equation (20) is given by

$$
a\left(z_{1}\right)=\sqrt{\frac{2 b}{Q}} \operatorname{sech}\left[\sqrt{\frac{-b}{P}} z_{1}\right] \text {. }
$$

It is more appropriate to set $b=\left(2 u_{e} u_{c}-u_{e}^{2}\right) / 4 P$, where $u_{e}$ and $u_{c}$ are real numbers which respectively represent envelope and carrier wave velocities measured in units of $\AA$ per dimensionless time $\tau$ [25]. Hence, solution (18) can now be re-written in the form [25]

$$
\psi\left(z_{1}, t_{2}\right)=\sqrt{\frac{2 u_{e} u_{c}-u_{e}^{2}}{2 P Q}} \operatorname{sech}\left[\sqrt{\frac{u_{e}^{2}-2 u_{e} u_{c}}{4 P^{2}}} z_{1}\right] \mathrm{e}^{i\left[\frac{u_{e}^{2}-2 u_{e} u_{c}}{4 P}\right] t_{2}} .
$$

When the integration constant $C$ is nonzero, the single pulse solution (22) becomes very unstable and difficult to be sustained in the system. Consequently, Equation (12) now admits periodic solution of the form [18] [24]

$$
\psi\left(z_{1}, t_{2}\right)=a_{0} d n\left[L z_{1}, k\right] \mathrm{e}^{i \zeta t_{2}},
$$

where $d n$ is a Jacobi elliptic function of modulus $k(0 \leq k \leq 1)$,

$$
a_{0}=u_{e} \sqrt{\frac{1-2 \eta}{-2\left(2-k^{2}\right) P Q}}, L=a_{0} \sqrt{\frac{-Q}{2 P}}, \zeta=-P L^{2},
$$

and $\eta=u_{c} / u_{e}$, with $0 \leq \eta<0.5$. The solution of Equation (8) in the cubic limit (i.e. $\gamma=\sigma=F_{3}=J_{3}=G_{4}=H_{4}=K_{4}=0$ ), now gives

$$
V(z, \tau)=2 a_{0 \varepsilon} d n\left[L_{\varepsilon} z, k\right] \cos (\Omega \tau)+2 a_{0 \varepsilon}^{2} d n^{2}\left[L_{\varepsilon} z, k\right]\left\{\frac{\alpha}{2}-\frac{\alpha}{3} \cos (2 \Omega \tau)\right\}+\bigcirc\left(\varepsilon^{3}\right),
$$

where

$$
a_{0 \varepsilon}=\varepsilon u_{e} \sqrt{\frac{1-2 \eta}{-2\left(2-k^{2}\right) P Q}}, L_{e}=\varepsilon a_{0 \varepsilon} \sqrt{\frac{-Q}{2 P}}, \zeta_{\varepsilon}=-P L_{\varepsilon}^{2}, \Omega=\omega+\varepsilon^{2} \zeta_{\varepsilon}, t_{0}=\tau .
$$

The constants $a_{0 \varepsilon}, 1 / L_{e}$, and $\Omega$, are respectively the amplitude, width, and angular frequency of the soliton solution that mimics the DNA open state configuration in the cubic limit. They are all dependent on the perturbation parameter $\varepsilon$, elliptic modulus $k$, and the experimental parameters of the DNA system as depicted in the contour plot in Figure 1. The amplitude $a_{0 \varepsilon}$ increases with increase in $\varepsilon$, and more appreciable for $\varepsilon>0.7$ and $k>0.8$. The width of the soliton $1 / L_{e}$, is measured in $\AA$ and more feasible for $\varepsilon>0.7$, irrespective of the values of $k$. Lastly, an increase in $\varepsilon$ generally diminishes the angular frequency $\Omega$, of the soliton. Based on the contour plot in Figure 1, we will 

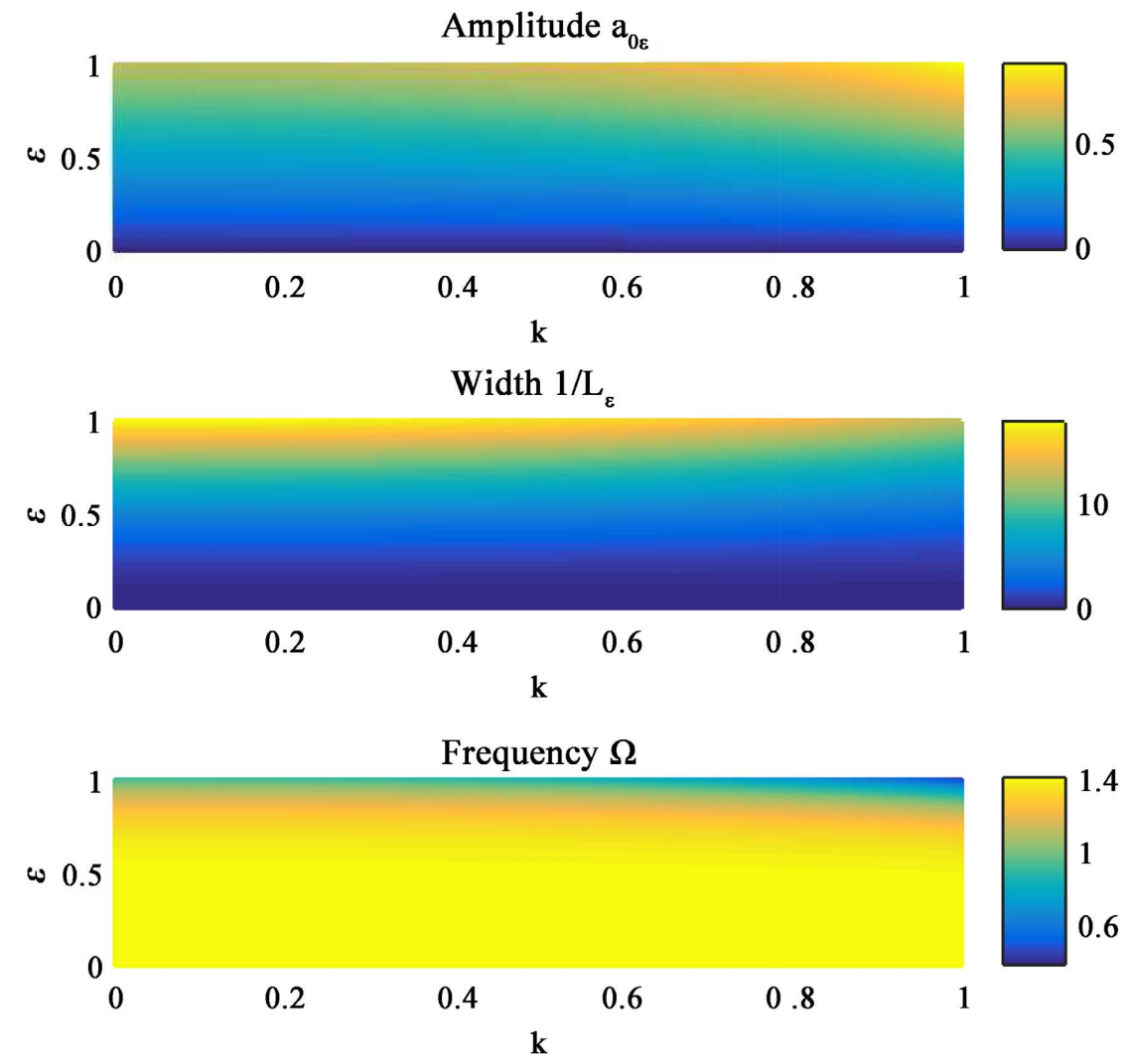

Figure 1. (color online) Variations of $a_{0 \varepsilon}, 1 / L_{e}$, and $\Omega$, with elliptic modulus modulus $k$ and perturbation parameter $\varepsilon$. The experimental values of the constants are $m=300 \mathrm{amu}$, $D_{0}=0.04 \mathrm{eV}, \quad a=4.45 \AA^{-1}, \quad \Delta H_{n}=0.44 \mathrm{eV}, \quad b=0.10 \AA^{-2}, \quad K_{b}=10^{-5} \mathrm{eV} \cdot \AA^{-2}$, $r=3.4 \AA, \omega=\sqrt{2}, C=2.00, u_{e}=1.00 \AA, u_{c}=0.00 \AA, \alpha=3 / 2$, and $\beta=-7 / 6$.

henceforth adopt $\varepsilon=1.00, k>0.8$, without loss of generality. The choice of the values of $\varepsilon$ and $k$, coupled with the experimental parameters given in Figure 1 , is best suited for us to analytically describe the base pair stretching that characterize the open state.

Figure 2 depicts the initial stages of the base pairs stretching in the cubic limit, which represent the breathing modes in the DNA molecular chain. We observe spatial periodic modes of the DNA base pairs stretching in Figure 2(a), for $k=0.88$, and Figure 2(b), for $k=0.98$. However for $k=1.00$, a more localized open state mode is observed in Figure 2(c); similar to the higher order discrete breather mode [26], and spatial compaction profile [39]. The spatiotemporal profile in Figure 2 generally portrays a very stable structural wave features, probably because it is still evolving at the early stages (i.e. $\tau$ varies from 0.00 to 60.0).

Concretely at the initial stage for $k=0.88$, and $k=0.98$, as in Figure 3(a) and Figure 3 (b) respectively, the breathing modes of the DNA is purely periodic. These modes degenerate to a strong secant hyperbolic excitation in Figure 3 (c) for $k=1.00$. These excitations mainly mimics the open state configuration, and a precursor for the transcription, replication, and transmission of genetic codes 
(a)

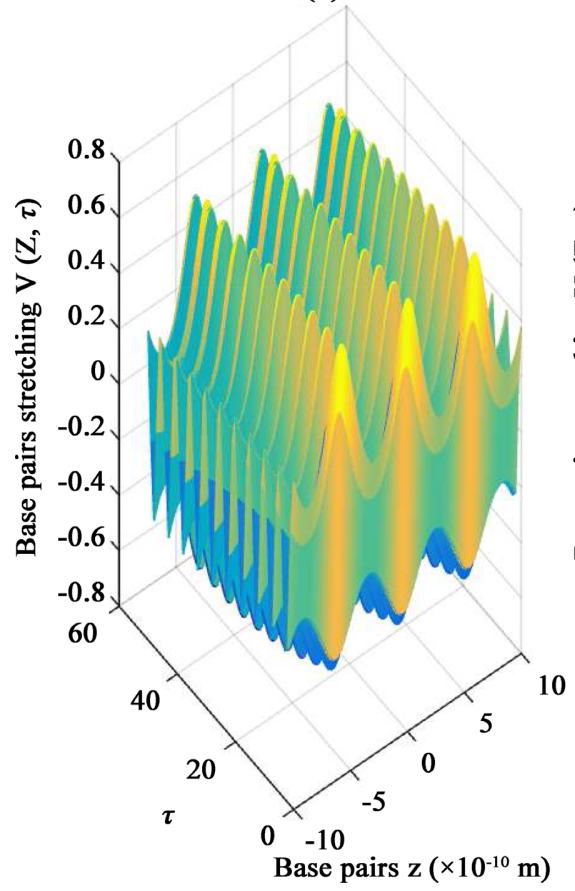

(b)

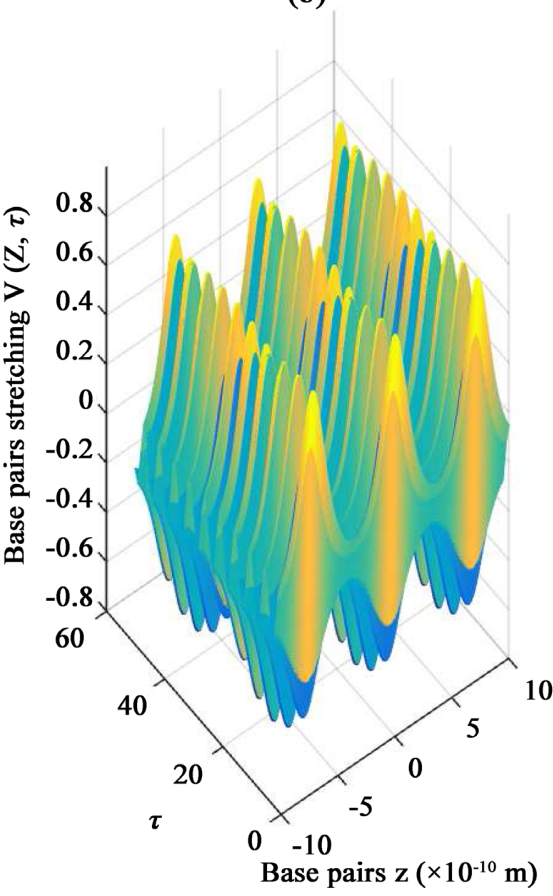

(c)

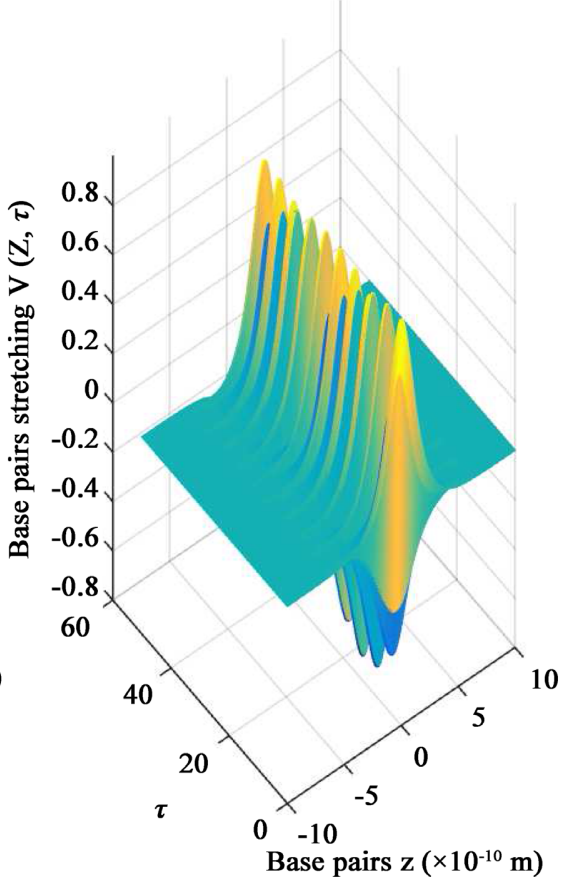

Figure 2. (color online) Three dimensional plot at the initial stages of base pairs stretching, according to solution (25). Parameters are: $\quad m=300 \mathrm{amu}, \quad D_{0}=0.04 \mathrm{eV}, \quad a=4.45 \AA^{-1}, \quad \Delta H_{n}=0.44 \mathrm{eV}, \quad b=0.10 \AA^{-2}, \quad K_{b}=10^{-5} \mathrm{eV} \cdot \AA^{-2}, \quad r=3.4 \AA, \quad \omega=\sqrt{2}$, $C=2.00, u_{e}=1.00 \AA, u_{c}=0.40 \AA, \alpha=3 / 2, \beta=-7 / 6$, and $\varepsilon=1.00$. This is for elliptic modulus: (a) $k=0.88$, (b) $k=0.98,(\mathrm{~d}) \quad k=1.00$.

in the DNA double strand chain [1]-[8]. After a period of time $t=100 \mathrm{ps}$ $(\tau=505.05)$, the modes in Figures 3(a)-(c) gradually changes to give the profile shown in Figures 3(d)-(f); where the nucleotide stretching becomes more pronounced especially as in Figure 3(f) for $k=1.00$. The base pairs stretching which is governed by the analytical solution (25), equally evolve to give a structurally robust breather modes in Figures $3(\mathrm{~g})$-(i) after $t=200 \mathrm{ps} \quad(\tau=1010.10)$.

We further plot the long-time evolution of base pair stretching, at different times as shown in Figure 4. The periodic and localized modes in Figure 4, are also known as the DNA fluctuational opening; best described as precursor states for the local denaturation observed during DNA transcription. It equally captures the thermal denaturation process, based on the finite stacking enthalpy DNA model. The structural variations of the nucleotide base pairs stretching in Figure 3 and Figure 4, simply points to the instability of the DNA open states. Consequently, we will carry out a linear stability analysis in the proceeding subsection in order to test the robustness of these spatial periodic DNA modes.

\subsection{Stability Analysis}

In the preceding subsection, we obtained localized periodic wave trains that mimic base pairs stretching in the finite stacking enthalpy DNA molecular chain. To discuss the stability of these periodic breathing modes, one must superimpose a small perturbation on this solution and analyze the evolution of the 

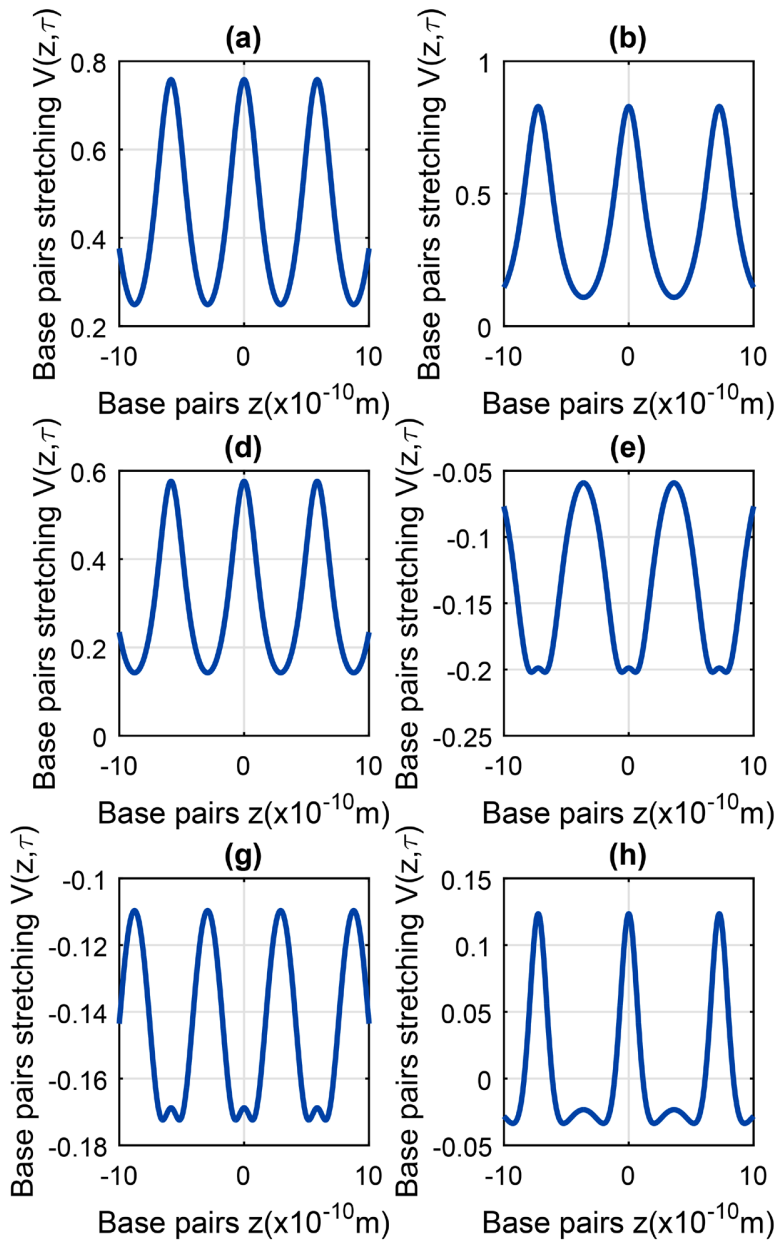

Base pairs $z\left(\times 10^{-10} \mathrm{~m}\right)$
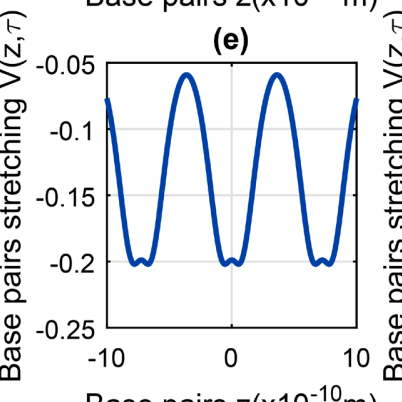

Base pairs $\mathrm{z}\left(\times 10^{-10} \mathrm{~m}\right)$

(h)

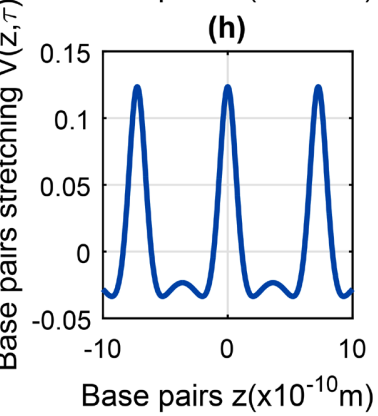

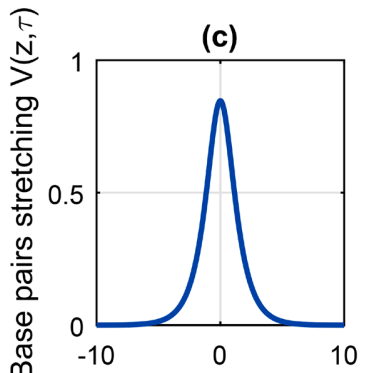

Base pairs $z\left(\times 10^{-10} \mathrm{~m}\right)$

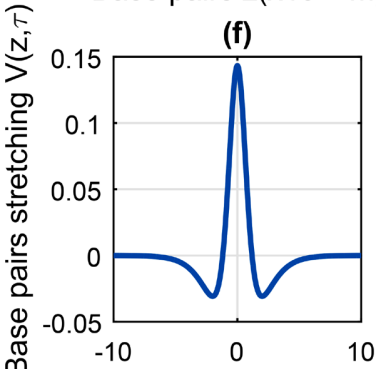

Base pairs $\mathrm{z}\left(\times 10^{-10} \mathrm{~m}\right)$

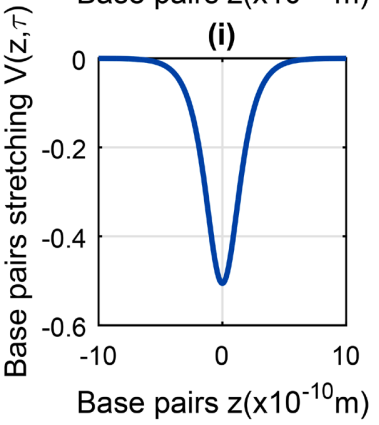

Figure 3. Base pairs stretching according to solution (25), for experimental values: $m=300 \mathrm{amu}, D_{0}=0.04 \mathrm{eV}, \quad a=4.45 \AA^{-1}, \Delta H_{n}=0.44 \mathrm{eV}, \quad b=0.10 \AA^{-2}$,

$K_{b}=10^{-5} \mathrm{eV} \cdot \AA^{-2}, r=3.4 \AA, \omega=\sqrt{2}, C=2.00, u_{e}=1.00 \AA, u_{c}=0.40 \AA, \alpha=3 / 2$, $\beta=-7 / 6$, and $\varepsilon=1.00$. This is for dimensionless time $(\tau)$ and elliptic modulus $(k)$, as follows: (a) $\tau=0.00, k=0.88$, (b) $\tau=0.00, k=0.98$, (c) $\tau=0.00, k=1.00$, (d) $\tau=505.05, \quad k=0.88$, (e) $\tau=505.05, k=0.98$, (f) $\tau=505.05, k=1.00, \quad$ (g) $\tau=1010.10, k=0.88$, (h) $\tau=1010.10, k=0.98$, (i) $\tau=1010.10, k=1.00$.

perturbation. Note that stability analysis is an important issue related to the study of nonlinear dynamical systems because it provides an effective way of testing the robustness of the soliton trains against small perturbation in the amplitude. Stability analysis is applied in a diverse manner, based on the complexity of the physical system under review and the type of solution involved.

In order to investigate the linear stability of the spatial periodic soliton mode, we consider small perturbations $a_{p}\left(z_{1}\right)$ to the amplitude of the DNA excitation mode denoted by $a_{0}\left(z_{1}\right)$, so that

$$
\psi\left(z_{1}, t_{2}\right)=\left[a_{0}\left(z_{1}\right)+\varepsilon a_{p}\left(z_{1}\right)\right] \mathrm{e}^{i b t_{2}},
$$

where $\varepsilon \ll 1$. After the nonlinear interactions, the resultant internal modes of vibration carrying the genetic codes is obtained by substituting Equation (27) into Equation (12) and considering terms to the various orders of $\varepsilon$ : 

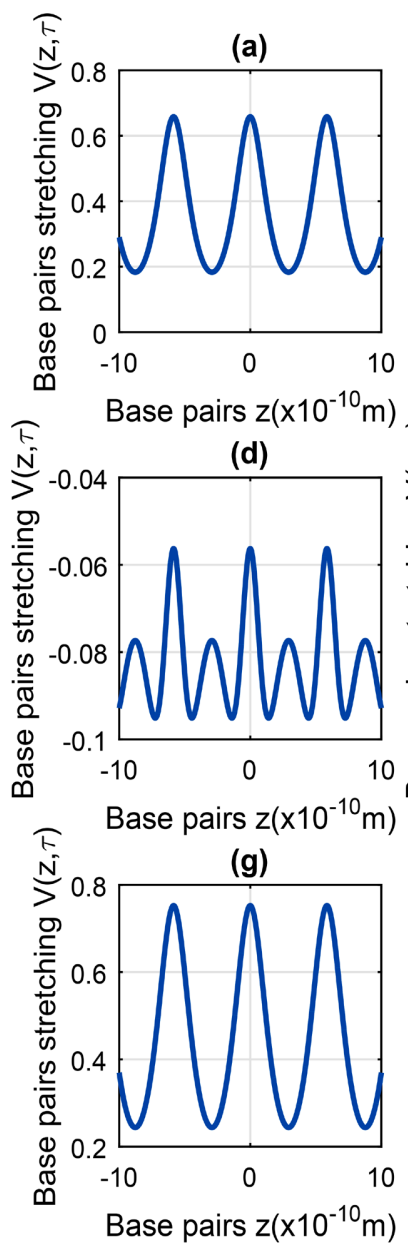
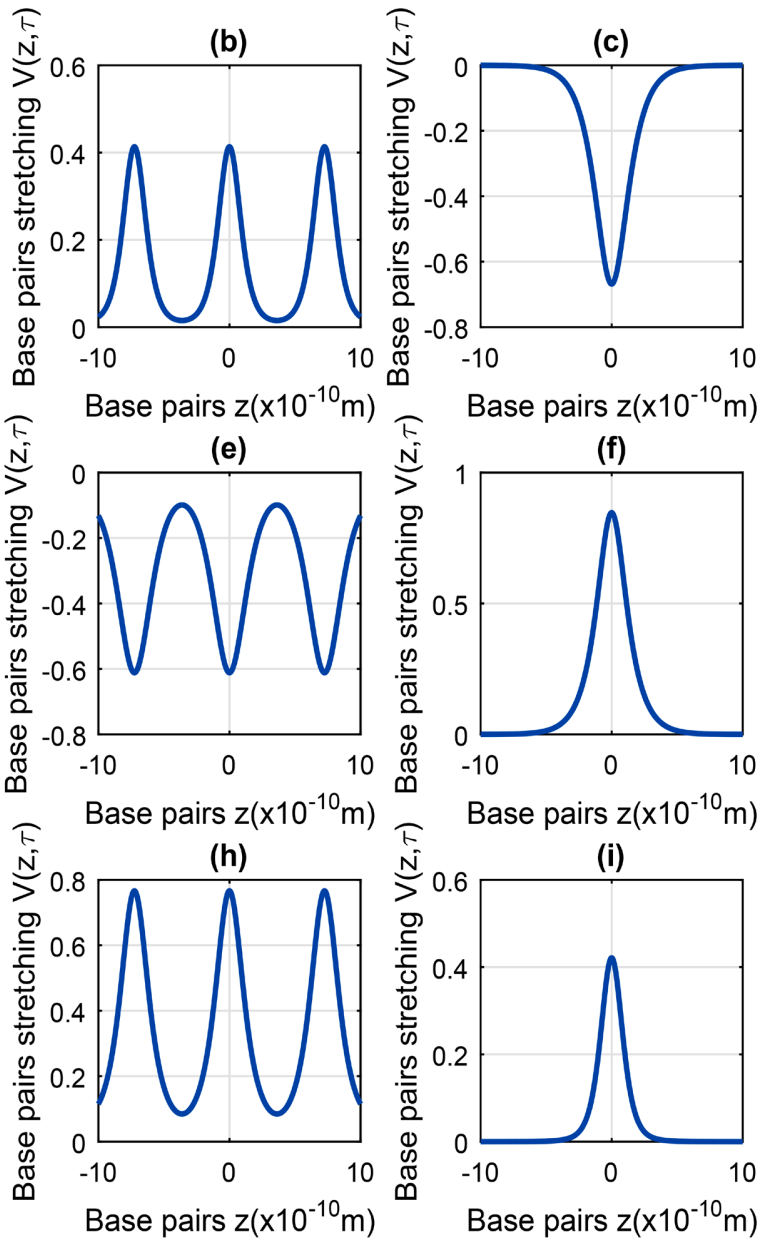

(i)

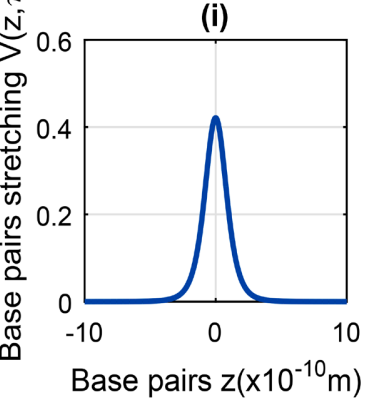

Figure 4. Parameters are the same as in Figure 3, but for: (a) $\tau=2525.25, k=0.88$, (b) $\tau=2525.25, k=0.98$, (c) $\tau=2525.25, k=1.00$, (d) $\tau=2700.00, k=0.88$, (e) $\tau=2700.00, k=0.98$, (f) $\tau=2700.00, k=1.00$, (g) $\tau=3030.30, k=0.88$, (h) $\tau=3030.30, k=0.98$, (i) $\tau=3030.30, k=1.00$.

Order $\varepsilon^{0}$,

$$
P \frac{\mathrm{d}^{2} a_{0}}{\mathrm{~d} z_{1}^{2}}-Q a_{0}^{3}+b a_{0}=0
$$

Order $\varepsilon^{1}$

$$
P \frac{\mathrm{d}^{2} a_{p}}{\mathrm{~d} z_{1}^{2}}-3 Q a_{0}^{2} a_{p}+b a_{p}=0 .
$$

We have already obtained periodic solution of Equation (28) as

$$
a_{0}\left(z_{1}\right)=\sqrt{\frac{2 b}{Q\left(2-k^{2}\right)}} d n\left[\sqrt{\frac{-b}{P\left(2-k^{2}\right)}} z_{1}, k\right],
$$

which can be substituted into Equation (29) and simplified to obtain [18] [24]

$$
\frac{\mathrm{d}^{2} a_{p}}{\mathrm{~d} \xi^{2}}+\left[E(k)-6 k^{2} \operatorname{sn}^{2}(\xi, k)\right] a_{p}=0,
$$


where $\lambda=\sqrt{\frac{-b}{P\left(2-k^{2}\right)}}, \xi=\lambda z_{1}$, and $E(k)=\frac{6 P \lambda^{2}+b}{P \lambda^{2}}$. Equation (31) is known as the Lamé equation of the second order with five distinct localized solutions [18] [24]

$$
\begin{gathered}
a_{p 1}(\xi)=A_{1}(k) c n(\xi, k) d n(\xi, k), b_{1}=\left(5-k^{2}\right)|P| \lambda^{2}, \\
a_{p 2}(\xi)=A_{2}(k) c n(\xi, k) \operatorname{sn}(\xi, k), b_{2}=\left(2-k^{2}\right)|P| \lambda^{2}, \\
a_{p 3}(\xi)=A_{3}(k) \operatorname{sn}(\xi, k) d n(\xi, k), b_{3}=\left(5-4 k^{2}\right)|P| \lambda^{2}, \\
a_{p 4, p 5}(\xi)=A_{4,5}(k)\left[s n^{2}(\xi, k)-\frac{1+k^{2}}{3 k^{2}} \mp \frac{\sqrt{1-k^{2}\left(1-k^{2}\right)}}{3 k^{2}}\right], \\
b_{4,5}=\left[2-k^{2} \pm \frac{\sqrt{1-k^{2}\left(1-k^{2}\right)}}{2}\right]|P| \lambda^{2} .
\end{gathered}
$$

The amplitudes $A_{i}(k)$ for $i=1,2,3,4,5$ can be obtained by using appropriate orthogonality and normalization relations [45], and the profile of these perturbations are given in Figure 5. The spatial period and length of the perturbed solitons $a_{p i}(\xi)$, is controlled by the elliptic modulus $k$. In fact for $k=1.00$, the separation between solitons become infinite and periodic train degenerates to a localized mode. From the profiles of these single solitons on the right column in Figure 5, it is clear that the distinct localized soliton modes are both symmetric and asymmetric with respect to the origin.

The five bound states given in Figure 5 shows the various genetic code structures, that clearly signifies the complex nature of the transcription and replication processes. Gene transfer under appropriate physiological conditions is indeed possible only in one of these modes. Concretely from the standpoint of biophysics, these localized modes describe internal oscillations in the DNA structure which is capable of exposing all hidden modes that may be responsible for gene mutations or other physiological disorders [25] [42]. These bound states can also be associated with radiation-carrying excitations in the background of the DNA open state, by virtue of their nonzero energies [18].

\section{Higher Order Stretching of Nucleotide Base Pairs}

In order to give a comprehensive account of the base pairs stretching that mimics the DNA open state, we now incorporate all the terms in the ansatz (10). However we set $F_{3}=0$, without of loss of generality, since we are dealing with periodic modes. The term $F_{3}$ generally introduces secularity which makes the solution cumbersome, and blurs the basic physics that characterize periodic open state dynamics of finite stacking enthalpy DNA. It is important to note that in the investigation of stationary breather modes of generalized nonlinear Klein-Gordon lattices, $F_{3} \neq 0 \quad$ [44].

Simplification of Equations (13) to (19), naturally lead us to the following 
(a)

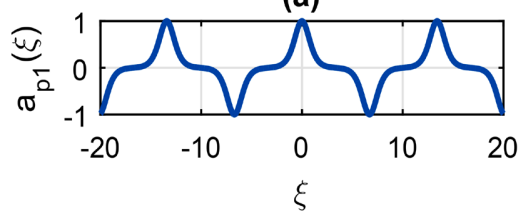

(c)

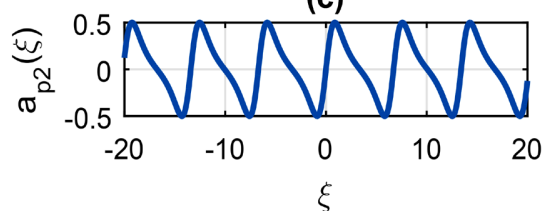

(e)

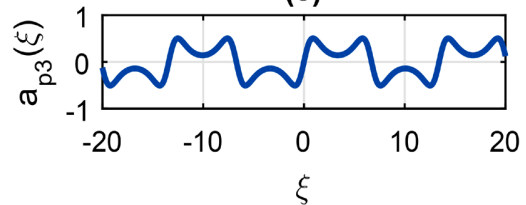

(g)

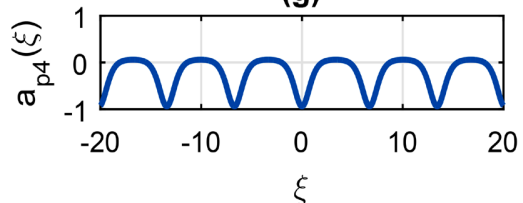

(i)

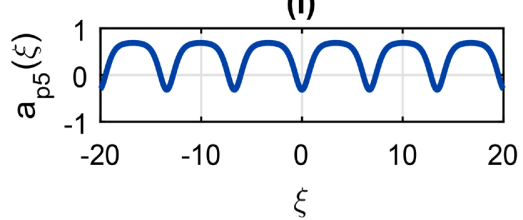

(b)

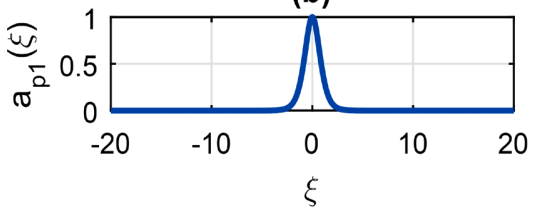

(d)

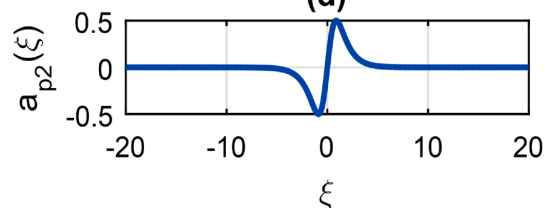

(f)

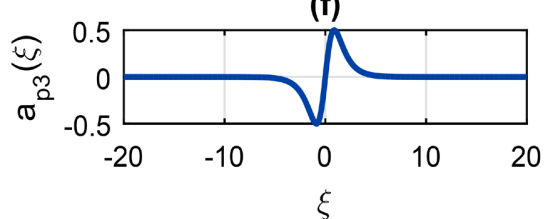

(h)

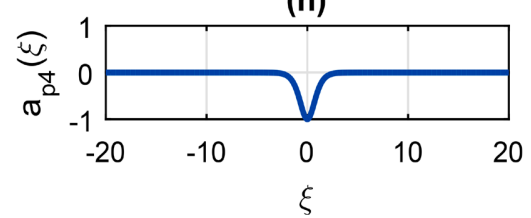

(j)

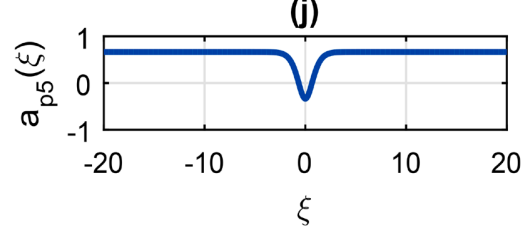

Figure 5. Profiles of bound state solutions (32), of the second order Lamé Equation (31) with $A_{i}(k)=1.0$, for $i=1,2,3,4,5$. The left column is for $k=0.98$, while the right is for $k=1.00$.

important quantities:

$$
\begin{gathered}
J_{3}=\frac{1}{24}\left[2 \alpha^{2}-3 \beta\right] \psi^{3}, \\
G_{4}=\frac{K_{0} \alpha}{\omega^{2}}\left[\frac{\partial^{2}|\psi|^{2}}{\partial z_{1}^{2}}\right]+\left[3 \gamma+5 \alpha \beta+\frac{19}{9} \alpha^{3}\right]|\psi|^{4}, \\
H_{4}=\frac{2 K_{0} \alpha}{9 \omega^{2}}\left[\frac{\partial \psi^{2}}{\partial z_{1}}-\psi \frac{\partial^{2} \psi}{\partial z_{1}^{2}}\right]-\left[\frac{4}{3} \gamma+\frac{31}{12} \alpha \beta+\frac{59}{54} \alpha^{3}\right]|\psi|^{2} \psi^{2}, \\
K_{4}=\left[\frac{1}{12} \alpha \beta-\frac{1}{15} \gamma-\frac{1}{54} \alpha^{3}\right] \psi^{4} .
\end{gathered}
$$

Consequently, Equation (17) can now be re-written as

$$
\begin{aligned}
2 i \omega \frac{\partial \psi}{\partial t_{4}}+\frac{\partial^{2} \psi}{\partial t_{2}^{2}}= & 2 K_{0} \frac{\partial^{2} \psi}{\partial z_{1} \partial z_{3}}+4 K_{0} \alpha^{2} \psi \frac{\partial^{2}|\psi|^{2}}{\partial z_{1}^{2}}+\frac{2 K_{0} \alpha}{\omega^{2}}\left[\psi^{*} \frac{\partial \psi^{2}}{\partial z_{1}}-|\psi|^{2} \frac{\partial^{2} \psi}{\partial z_{1}^{2}}\right] \\
& +\omega^{2}\left[\frac{84}{3} \alpha \gamma+\frac{635}{36} \alpha^{2} \beta-\frac{1005}{162} \alpha^{4}-\frac{3}{8} \beta^{2}+10 \sigma\right] \psi|\psi|^{4}
\end{aligned}
$$


Based on the values obtained for $J_{3}, G_{4}, H_{4}, K_{4}$, we can conveniently modify solution (23) to now read [44]

$$
\psi\left(z_{1}, t_{2}, z_{3}, t_{4}\right)=a_{0} d n\left[L z_{1}+L_{3} z_{3}, k\right] \mathrm{e}^{i\left(\zeta t_{2}+\zeta_{4} t_{4}\right)},
$$

where $L_{3}$ and $\zeta_{4}$ are real constants. To determine the values of $L_{3}$ and $\zeta_{4}$, we substitute the modified solution (35) into Equation (34) and group coefficients of terms of the orders $d n^{0}[], d n^{2}[]$ and so on. After some rigorous calculations and simplifications, this leads us to

$$
\begin{gathered}
L_{3}=L a_{0}^{2} \alpha\left[4 \alpha\left(3-2 k^{2}\right)+\frac{1}{\omega}\left(k^{2}-1\right)\right], \\
\zeta_{4}=\frac{K_{0} \alpha L^{2} a_{0}^{2}\left(k^{2}-1\right)^{2}\left[1-4 \alpha \omega^{2}\left(4-k^{2}\right)\right]}{\omega^{3}}-\frac{\zeta}{2 \omega} .
\end{gathered}
$$

The most appropriate form of the analytic solution of the higher order stretching of nucleotide base pairs is now obtained by substituting the solution (35), into the ansatz (10) to have

$$
\begin{aligned}
& V(z, \tau)=2 a_{0 \varepsilon}^{\prime} d n\left[L_{\varepsilon}^{\prime} z, k\right] \cos \left(\Omega^{\prime} \tau\right)+a_{0 \varepsilon}^{\prime 2} d n^{2}\left[L_{\varepsilon}^{\prime} z, k\right]\left\{\frac{3}{2}-\cos \left(2 \Omega^{\prime} \tau\right)\right\} \\
& +\frac{2}{3} a_{0 \varepsilon}^{\prime 3} d n^{3}\left[L_{\varepsilon}^{\prime} z, k\right] \cos \left(3 \Omega^{\prime} \tau\right)-a_{0 \varepsilon}^{\prime 4} d n^{4}\left[L_{\varepsilon}^{\prime} z, k\right]\left\{\frac{1}{2}+\frac{23}{50} \cos \left(4 \Omega^{\prime} \tau\right)\right\} \\
& +\frac{33}{50} \varepsilon a_{0 \varepsilon}^{\prime 3} d n^{3}\left[L_{\varepsilon}^{\prime} z, k\right] \cos \left(\left[3 \Omega^{\prime}-\sqrt{2}\right] \tau\right)+3 \varepsilon^{2} K_{0} L^{2} a_{0 \varepsilon}^{\prime 2} d n\left[L_{\varepsilon}^{\prime} z, k\right]\left\{d n^{3}\left[L_{\varepsilon}^{\prime} z, k\right]\right. \\
& \left.+\left(k^{2}-1\right) d n\left(L_{\varepsilon}^{\prime} z, k\right)-\frac{2}{L} k^{2} \operatorname{sn}\left[L_{\varepsilon}^{\prime} z, k\right] c n\left[L_{\varepsilon}^{\prime} z, k\right]\right\} \cos \left(2 \Omega^{\prime} \tau\right) \\
& +\frac{3}{2} \varepsilon^{2} K_{0} L^{2} a_{0 \varepsilon}^{\prime 2} d n\left[L_{\varepsilon}^{\prime} z, k\right]\left\{k^{2}-1+2\left(1-k^{2}\right) d n^{2}\left[L_{\varepsilon}^{\prime} z, k\right]-2 d n^{4}\left[L_{\varepsilon}^{\prime} z, k\right]\right\} .
\end{aligned}
$$

The constants in solution (37) are

$$
\begin{gathered}
a_{0 \varepsilon}^{\prime}=\varepsilon u_{e} \sqrt{\frac{1-2 \eta}{-2\left(2-k^{2}\right) P Q}}, L_{\varepsilon}^{\prime}=\varepsilon a_{0 \varepsilon}^{\prime} \sqrt{\frac{-Q}{2 P}}\left\{1+\varepsilon^{2} a_{0 \varepsilon}^{\prime 2}\left(25.9-16.9 k^{2}\right)\right\}, \\
\Omega^{\prime}=\sqrt{2}+0.325 \varepsilon^{4} Q a_{0 \varepsilon}^{\prime 2}+0.53 \varepsilon^{4} K_{0} L^{2} a_{0}^{2}\left(k^{2}-1\right)^{2}\left(12 k^{2}-47\right),
\end{gathered}
$$

and the values of $L$ and $a_{0}$ are given in Equation (24).

The profiles of the higher order modes are given in Figure 6. It clearly depicts the stretching of DNA double strand. Such open states lead to a better representation of the base pairs stretching that generally precedes the transcription and replication processes. Furthermore, it equally depicts a more accurate energy activator for RNA-polymerase transport during the periodic opening of DNA double strand chain, thereby exposing more bases out of the stack. As shown in (Figure 6(a), Figure 6(d), Figure 6(g)) for $k=0.88$, and (Figure 6(b), Figure 6(e), Figure $6(\mathrm{~h}))$ for $k=0.98$, we observe that more base pairs are experiencing a very structurally stable open state configurations. However for $k=1.00$ as in (Figure 6(c), Figure 6(f), Figure 6(i)), only few base pairs open up during the transcription process as experimentally confirmed with DNA double helix 

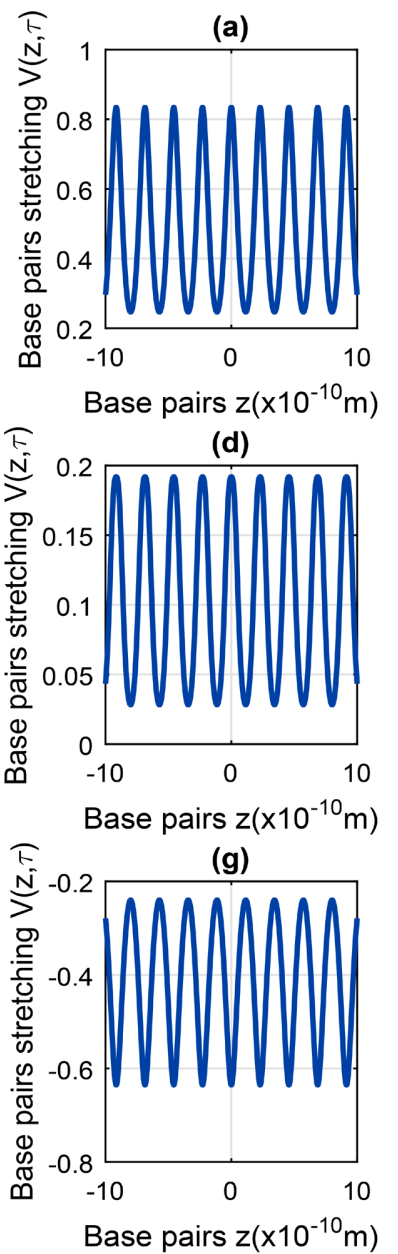

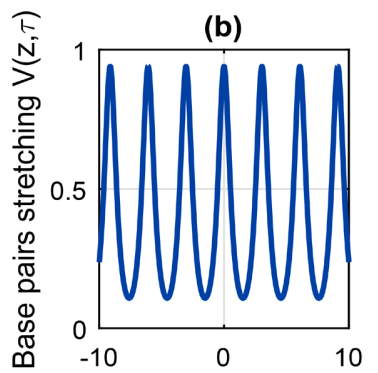

Base pairs $z\left(\times 10^{-10} \mathrm{~m}\right)$

(e)

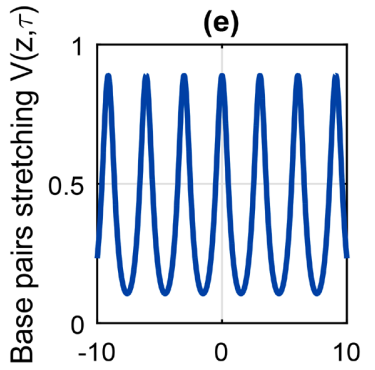

Base pairs $z\left(\times 10^{-10} \mathrm{~m}\right)$

(h)

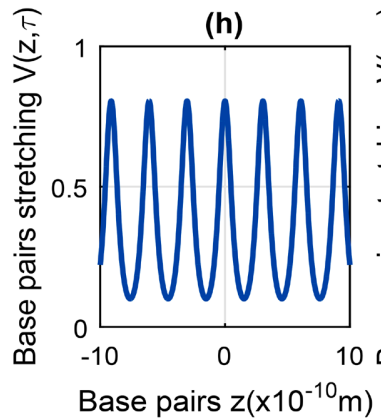

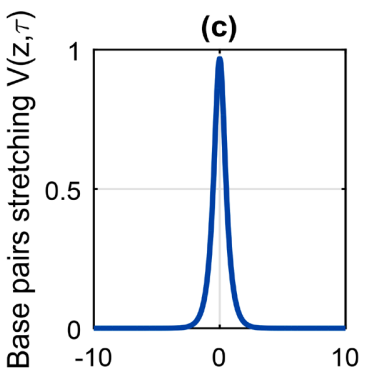

Base pairs $\mathrm{z}\left(\times 10^{-10} \mathrm{~m}\right)$

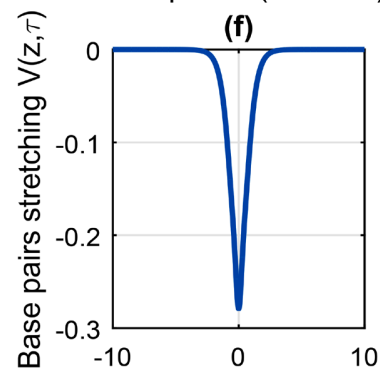

Base pairs $z\left(\times 10^{-10} \mathrm{~m}\right)$

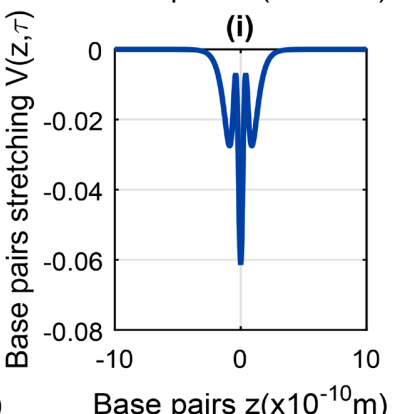

Figure 6. Higher order base pairs stretching according to solution (37), for experimental values: $m=300 \mathrm{amu}, \quad D_{0}=0.04 \mathrm{eV}, \quad a=4.45 \AA^{-1}, \Delta H_{n}=0.44 \mathrm{eV}, \quad b=0.10 \AA^{-2}$, $K_{b}=10^{-5} \mathrm{eV} \cdot \AA^{-2}, r=3.4 \AA, \omega=\sqrt{2}, C=2.00, u_{e}=1.00 \AA, u_{c}=0.40 \AA, \alpha=3 / 2$, $\beta=-7 / 6, \gamma=5 / 8, \sigma=-31 / 120$, and $\varepsilon=1.00$. This is for: (a) $\tau=0.00, k=0.88$, (b) $\tau=0.00, k=0.98$, (c) $\tau=0.00, k=1.00$, (d) $\tau=505.05, k=0.88$, (e) $\tau=505.05, \quad k=0.98$, (f) $\tau=505.05, k=1.00$, (g) $\tau=1010.10, k=0.88$, (h) $\tau=1010.10, k=0.98$, (i) $\tau=1010.10, k=1.00$.

[46] [47]. This mainly confirms that the soliton solution (37), gives a more accurate analytic representation within theoretical limits [48]; of stable periodic open states under appropriate physiological conditions.

The long time evolution of the gradual unzipping of the DNA molecule is captured in Figure 7, which is characterized by minimal distortion of the periodic modes. It is important to note that the stretching of the base pairs in Figure 6(i) and Figure 7(c), Figure 7(f) for $k=1.00$, induces minor distortions in the open state by the slight splitting of a single pulse. This may have long term effects during the transcription and replication processes, as the open state becomes more susceptible to external attack. Such attacks may alter some parameters of the DNA system, hence distorting the reading of genetic codes and induces gene mutations which is responsible for numerous diseases [25] [42] [46] 

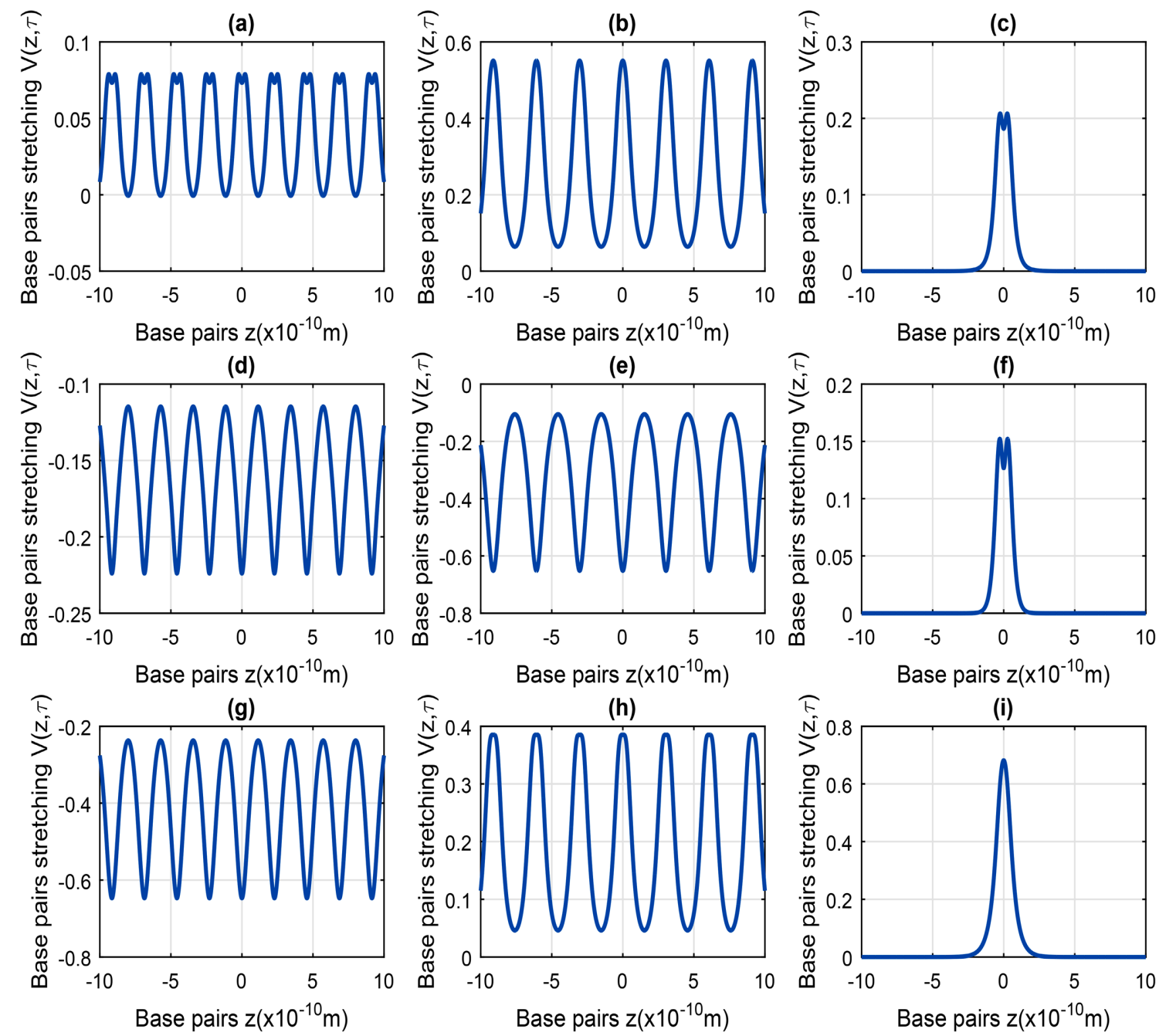

Figure 7. Parameters are the same as in Figure 6, but for: (a) $\tau=2525.25, k=0.88$, (b) $\tau=2525.25, k=0.98$, (c) $\tau=2525.25, k=1.00$, (d) $\tau=2700.00, k=0.88$, (e) $\tau=2700.00, k=0.98$, (f) $\tau=2700.00, k=1.00$, (g) $\tau=3030.30, k=0.88$, (h) $\tau=3030.30, k=0.98$, (i) $\tau=3030.30, k=1.00$.

[47]. A careful observation shows that the structural stability and number of solitons in the base pairs stretching of Figure 6 and Figure 7, supersedes that of their cubic limit counterparts in Figure 3 and Figure 4 respectively. Since all the experimental values used are the same, the comparison strongly suggest that the higher order modes solution (37), reflects a more realistic open state configuration during the transcription and replication processes.

\section{Discussion and Conclusion}

The nucleotide is an elementary unit which consists of sugar, phosphate, and base. The segment of a DNA which is responsible for biosynthesis of a single polypeptide chain is called a gene, which averagely contains about 900 to 1500 nucleotide base pairs. During the transcription and replication processes, these genes are effectively transferred to single stranded shorter RNA. In fact, bases freely interact with enzymes during the local opening of DNA chain and there- 
fore play a key regulatory role in the transcription process. This study seeks to expose the various forms of periodic base pairs stretching of DNA, in order to elucidate on intrinsic processes like transmission, transcription, and replication of genetic codes. Concretely, the DNA remains the basic organ of a cell which stores all vital information that ensures the effective growth and reproduction of all living organisms. Some distorted profile of base pairs stretching identified in this study may make the system so vulnerable to external attack, and hinders the smooth flow of genetic codes.

We effectively derived higher order nonlinear Schrödinger amplitude equations from the BJ model of DNA, by using the extended multiple scale asymptotic methods. Periodic solutions of these amplitude equations were used to mimic the open state configurations of a DNA strand under appropriate physiological conditions. The BJ model can also provide valuable information relating to the thermodynamic properties of DNA, by showing that the finite enthalpy may be responsible for the DNA denaturation. The stability analysis shows the existence of other background modes that may provide a possible physical mechanism for the effect of finite enthalpy stacking on DNA dynamics to be investigated. The stacking interaction of the BJ model equally provides both linear and nonlinear coupling parameters in the system which can independently control the dynamics and stability of the periodic solutions. We believe that this work opens up new vision on the concept of nonlinear periodic waves in DNA, and can also be exported in the study of many other physical systems.

In perspective, it will be very interesting to investigate on the impact of the helicoidal interactions on the periodic stretching of base pairs. The helicoidal term can best be appreciated because of the twisted nature of a real DNA molecule. Secondly, the interactions between the oscillating nucleotides and the aqueous environment naturally induce frictional forces, that must not be neglected in order to holistically describe the DNA dynamics. Lastly, quantum theory needs to be fully incorporated in order to comprehensively describe the DNA dynamics at the level much smaller than the nucleotides [49].

\section{Acknowledgements}

N. Oma Nfor appreciates the enriching discussions with S. Bruno Yamgoué, on the dynamics of DNA molecule.

\section{Conflicts of Interest}

The author declares no conflicts of interest regarding the publication of this paper.

\section{References}

[1] Englander, S.W., Kallenbach, N.R., Heeger, A.J., Krumhansl, J.A. and Litwin, S. (1980) Proceedings of the National Academy of Sciences of the United States of America, 77, 7222. https://doi.org/10.1073/pnas.77.12.7222 
[2] Joyeux, M. and Buyukdagli, S. (2005) Physical Review E, 72, Article ID: 051902. https://doi.org/10.1103/PhysRevE.72.051902

[3] Joyeux, M. and Buyukdagli, S. (2008) Physical Review E, 77, Article ID: 031903. https://doi.org/10.1103/PhysRevE.77.031903

[4] Joyeux, M. and Florescu, A.-M. (2009) Journal of Physics: Condensed Matter, 21, Article ID: 34101. https://doi.org/10.1088/0953-8984/21/3/034101

[5] Yakusevich, L. (2004) Nonlinear Physics of DNA. Wiley Series in Nonlinear Sciences. John Wiley and Sons, Weinheim.

[6] Dauxois, T., Peyrard, M. and Bishop, A.R. (1993) Physical Review E, 47, 684-695. https://doi.org/10.1103/PhysRevE.47.684

[7] Dauxois, T. and Peyrard, M. (1995) Physical Review E, 51, 4027-4040. https://doi.org/10.1103/PhysRevE.51.4027

[8] Peyrard, M. (2004) Nonlinearity, 17, R1-R40. https://doi.org/10.1088/0951-7715/17/2/R01

[9] El Kinani, R., Kaidi, H. and Barka, N. (2020) Open Journal of Biophysics, 10, 129-149. https://doi.org/10.4236/ojbiphy.2020.103011

[10] Biegeleisen, K. (2016) Open Access Library Journal, 3, 1-24. https://doi.org/10.4236/oalib.1103114

[11] Watson, J.D. (1976) Molecular Biology of the Gene. 3rd Edition, W. A. Benjamin, Menlo Park.

[12] Gotoh, O. (1983) Advances in Biophysics, 16, 3. https://doi.org/10.1016/0065-227X(83)90007-2

[13] Yakushevich, L.V., Savin, A.V. and Manevitch, L.I. (2002) Physical Review E, 66, Article ID: 016614. https://doi.org/10.1103/PhysRevE.66.016614

[14] Okaly, J.B., Ndzana, F.-II, Woulaché, R.L. and Kofané, T.C. (2019) The European Physical Journal Plus, 134, 598. https://doi.org/10.1140/epjp/i2019-12992-3

[15] Nfor, N.O., Yamgoué, S.B. and Moukam Kakmeni, F.M. (2021) Chinese Physics B. https://doi.org/10.1088/1674-1056/abbbf3

[16] Fermi, E., Pasta, J. and Ulam, S. (1965) Los Alamos Report LA-1940 (1955), Published Later. In: Segr, E., Ed., Collected Papers of Enrico Fermi, University of Chicago Press, Chicago.

[17] Nfor, N.O., Ghomsi, P.G. and Moukam Kakmeni, F.M. (2018) Physical Review E, 97, Article ID: 022214. https://doi.org/10.1103/PhysRevE.97.022214

[18] Achu, G.F., Mkam, S.E., Moukam Kakmeni, F.M. and Tchawoua, C. (2018) Physical Review E, 98, Article ID: 022216. https://doi.org/10.1103/PhysRevE.98.022216

[19] Nfor, N.O. and Mokoli, M.T. (2016) Journal of Modern Physics, 7, 1166-1180. https://doi.org/10.4236/jmp.2016.710106

[20] Achu, G.F., Moukam Kakmeni, F.M. and Dikandé, A.M. (2018) Physical Review E, 97, Article ID: 012211. https://doi.org/10.1103/PhysRevE.97.012211

[21] Moukam Kakmeni, F.M., Inack, E.M. and Yamakou, E.M. (2014) Physical Review E, 89, Article ID: 052919. https://doi.org/10.1103/PhysRevE.89.052919

[22] Agrawal, G.P. (2001) Nonlinear Fiber Optics. Third Edition, Academic Press, Cambridge.

[23] Kivshar, Y.S. and Agrawal, G.P. (2003) Optical Solitons: From Fibers to Photonic Crystals. Academic Press, San Diego. https://doi.org/10.1016/B978-012410590-4/50012-7 
[24] Fandio Jubgang, D.J., Dikandé, A.M. and Sunda-Meya, A. (2015) Physical Review $A$, 92, Article ID: 053850 . https://doi.org/10.1103/PhysRevA.92.053850

[25] Okaly, J.B., Mvogo, A., Tabi, C.B., Ekobena Fouda, H.P. and Kofané, T.C. (2020) Physical Review E, 102, Article ID: 062402. https://doi.org/10.1103/PhysRevE.102.062402

[26] Ying-Bo, Y., Xiao-Yun, W. and Bing, T. (2016) Journal of Biological Physics, 42, 213-222. https://doi.org/10.1007/s10867-015-9402-3

[27] Bang, O. and Peyrard, M. (1996) Physical Review E, 53, 4143. https://doi.org/10.1103/PhysRevE.53.4143

[28] Ting, J.L. and Peyrard, M. (1996) Physical Review E, 53, 1011.

[29] Englander, S.W., Kallenbach, N.R., Heeger, A.J., Krumhansl, J.A. and Litwin, S. (1980) Proceedings of the National Academy of Sciences of the United States of America, 77, 7222. https://doi.org/10.1073/pnas.77.12.7222

[30] Yakushevich, L.V. (1989) Physics Letters A, 136, 413-417. https://doi.org/10.1016/0375-9601(89)90425-8

[31] Yakushevich, L.V. (1994) Physica D, 79, 77-86. https://doi.org/10.1016/0167-2789(94)90038-8

[32] Gaeta, S. (2006) Physical Review E, 74, Article ID: 021921. https://doi.org/10.1103/PhysRevE.74.021921

[33] Gaeta G. (2007) Journal of Nonlinear Mathematical Physics, 14, 57-81. https://doi.org/10.2991/jnmp.2007.14.1.6

[34] Yomosa, S. (1983) Physical Review A, 27, 2120-2125. https://doi.org/10.1103/PhysRevA.27.2120

[35] Yomosa, S. (1984) Physical Review A, 30, 474-480. https://doi.org/10.1103/PhysRevA.30.474

[36] Homma, S. and Takeno, S. (1984) Progress of Theoretical Physics, 72, 679-693. https://doi.org/10.1143/PTP.72.679

[37] Campa, A. and Giansanti, A. (1998) Physical Review E, 58, 3585. https://doi.org/10.1103/PhysRevE.58.3585

[38] Gninzanlong, C.L., Ndjomatchoua, F.T. and Tchawoua, C. (2018) Chaos, 28, Article ID: 043105. https://doi.org/10.1063/1.5009147

[39] Ndjoko, P.B., Bilbault, J.M., Binczak, S. and Kofané, T.C. (2012) Physical Review E, 85, Article ID: 011916. https://doi.org/10.1103/PhysRevE.85.011916

[40] Vargas, E.V., Ludu, A., Hustert, R., Gumrich, P., Jackson, A.D. and Heimburg, T. (2011) Biophysical Chemistry, 153, 159. https://doi.org/10.1016/j.bpc.2010.11.001

[41] Nde Aboringong, E.N. and Dikandé, A.M. (2018) The European Physical Journal E, 41, 35. https://doi.org/10.1140/epje/i2018-11640-9

[42] Daniel, M. and Vasumathi, V. (2008) Physics Letters A, 372, 5144. https://doi.org/10.1016/j.physleta.2008.05.063

[43] Okaly, J.B., Ndzana, F.-II, Woulaché, R.L., Tabi, C.B. and Kofané, T.C. (2019) Chaos, 29, Article ID: 093103. https://doi.org/10.1063/1.5098341

[44] Jonathan, A.D.W. (1998) Journal of Physics A: Mathematical and General, 31, 3301-3323. https://doi.org/10.1088/0305-4470/31/14/016

[45] Dikandé, A.M. (1999) Physica Scripta, 60, 291. https://doi.org/10.1238/Physica.Regular.060a00291

[46] Plopper, G. (2012) Principles of Cell Biology. Jones and Bartlett Learning, Burling- 
ton.

[47] Balajee, A.S. and Phil, M. (2006) DNA Repair and Human Disease. Springer Science, New York.

[48] Fakhretdinov, M.I. and Zakiŕyanov, F.K. (2012) Russian Physics Journal, 54, 1304. https://doi.org/10.1007/s11182-012-9747-Z

[49] Chevizovich, D., Michieletto, D., Mvogo, A., Zakiryanov, F. and Zdravković, S. (2020) Royal Society Open Science, 7, Article ID: 200774.

https://doi.org/10.1098/rsos.200774 